\title{
Intraoperative computed tomography after tumor marking with metal clips for non-palpable lung tumors
}

\author{
Yoshimasa Tokunaga, Sung Soo Chang, Yusuke Kita, Taku Okamoto \\ Department of General Thoracic Surgery, Kochi Health Sciences Center, Kochi 781-8555, Japan \\ Contributions: (I) Conception and design: Y Tokunaga; (II) Administrative support: None; (III) Provision of study materials or patients: All authors; \\ (IV) Collection and assembly of data: All authors; (V) Data analysis and interpretation: Y Tokunaga; (VI) Manuscript writing: All authors; (VII) Final \\ approval of manuscript: All authors. \\ Correspondence to: Yoshimasa Tokunaga, MD, PhD. Department of General Thoracic Surgery, Kochi Health Sciences Center, 2125-1 Ike, Kochi \\ 781-8555, Japan. Email: yoshimasa.tokunaga@gmail.com.
}

\begin{abstract}
Background: Locating small, non-palpable lung tumors during video-assisted thoracoscopic surgery (VATS) is difficult. In this paper, we report a simple method to identify such tumors during VATS, using intraoperative computed tomography (IO-CT).

Methods: From 2015 to 2017, we performed IO-CT scans for patients who preoperatively seemed to have non-palpable lung tumors. We initially tried to locate these tumors by finger palpation through the thoracoscopic ports. IO-CT scans were performed after marking tumors with metal clips. However, difficultto-palpate tumors were marked by initially locating the intercostal muscle from preoperative CT. Metal clips were applied just under the intercostal muscle, and IO-CT scans were performed. After locating the tumor in relationship to the marking clips, patients would undergo wedge resections during VATS, using surgical staplers.

Results: We used this procedure on 21 tumors in 18 patients, including 9 non-palpable tumors and 12 palpable tumors (mean tumor size: $7.3 \mathrm{~mm}$; mean distance from pleura: $6.8 \mathrm{~mm}$ ). All tumors were identified intraoperatively, and all patients successfully underwent wedge resections during VATS, with no intrapostoperative complications.

Conclusion: IO-CT scans after tumor marking with metal clips during VATS can accurately locate nonpalpable small sized lung tumors. IO-CT scans should be indicated for tumors that are preoperatively considered to be non-palpable.
\end{abstract}

Keywords: Intraoperative computed tomography (IO-CT); metal clip marking; lung tumors; non-palpable

Submitted Feb 20, 2018. Accepted for publication Jun 15, 2018.

doi: $10.21037 /$ jtd.2018.06.123

View this article at: http://dx.doi.org/10.21037/jtd.2018.06.123

\section{Introduction}

Although computed tomography (CT) allows detection of small lung tumors, thoracic surgeons often need to resect small lung tumors that cannot be palpated preoperatively. Various tools, such as short hook wires, lipiodol and coils, are used to locate such tumors during surgery, particularly during video-assisted thoracoscopic surgery (VATS), but doing so is often difficult (1-3), and pneumothorax, intrapulmonary hematoma, hemothorax have reportedly been caused by these procedures; air embolism, particularly, can be fatal (4). New techniques have been developed to avoid such outcomes; for example, a bronchoscopic multispot dye-marking technique (virtual-assisted lung mapping; VAL-MAP), bronchoscopic metallic marking technique, ultrasonographic localization procedure, and the dyed swab method (5-8). However, these methods can be limited by tendencies for their landmark artifacts to be inaccurate or displaced, or their inappropriateness for many would-be patients. 
A


B

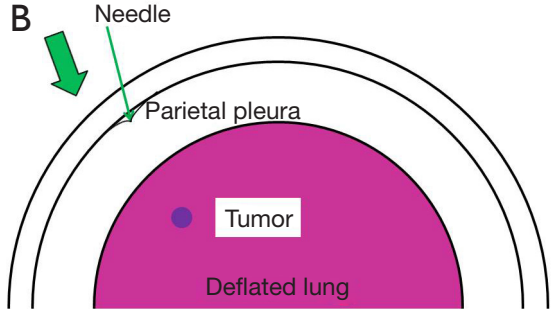

\begin{abstract}
E
\end{abstract}
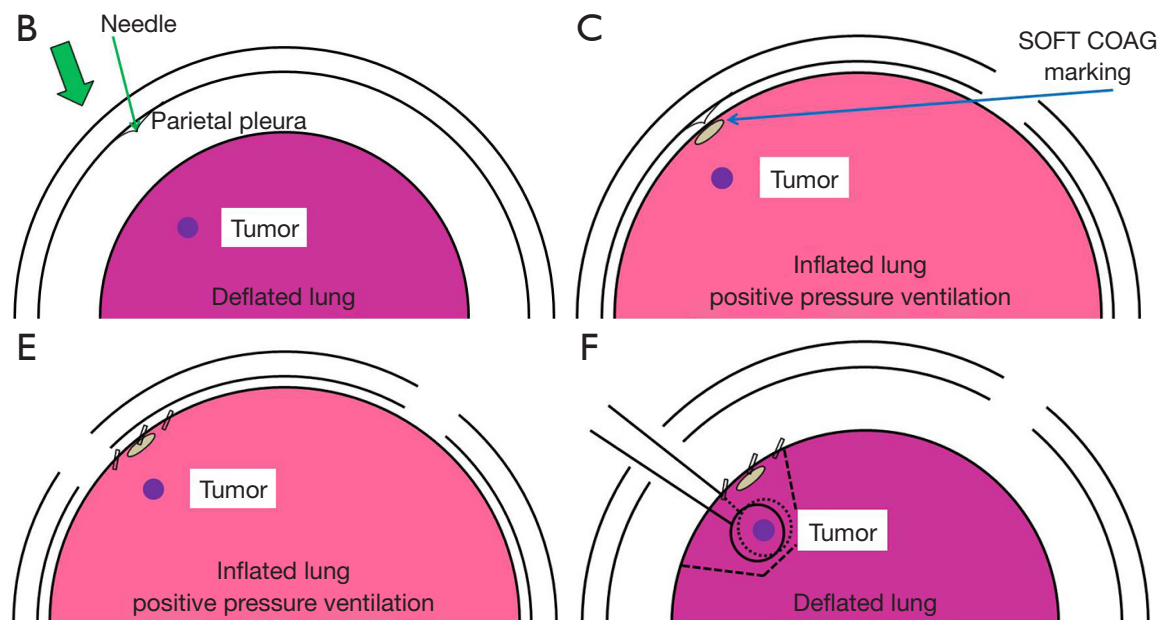

$\mathrm{F}$

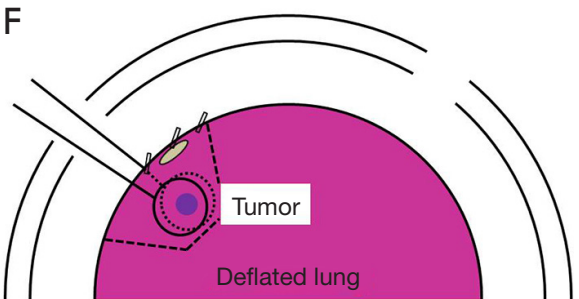

Figure 1 Marking difficult-to-palpate tumors by anatomical position. (A) Intercostal muscle site was estimated from preoperative CT; (B) needle is inserted from the intercostal muscle to make a depression in the parietal pleura; (C) under bilateral lung ventilation, the surface of the visceral pleura just under the needle depression is marked by SOFT COAG; (D) under unilateral lung ventilation again, metal clips are used to mark around the SOFT COAG marking; (E) intraoperative CT scans and 3D-CT imaging allow surgical team to locate the tumor by reference to the clips; $(\mathrm{F})$ the tumor was held by ring forceps; wedge resection was performed using surgical staplers.

In cardiac, vascular and neuro-surgery, hybrid operating rooms and intraoperative CT (IO-CT) scans are commonly used (Figure 1). Previously, we described how to establish anatomical intersegmental lines and obtain surgical margins with IO-CT scans during VATS segmentectomies (9). Because IO-CT scans can provide real-time lung images, we hypothesized that this procedure could precisely locate small non-palpable lung tumors. In this paper, we report a simple and feasible method to identify such lung tumors during VATS without special technique.

\section{Methods}

From January 2015 to December 2017, we performed IOCT scans for patients who preoperatively were thought to have non-palpable lung tumors. Palpation is considered difficult in cases where tumors have few solid components, are relatively far from the pleura, and/or have pleural adhesions. We excluded from this study patients who had undergone segmentectomy during VATS, as we had previously reported on their procedures (9). All tumors in the present study cohort required wedge resections during VATS, which were performed for the following reasons: multiple lung tumors, lung metastases from primary cancers in other organs, benign tumors, and low cardiopulmonary function. The study protocol was approved by the Institutional Review Board of Kochi Health Science
Center, Japan (No. 141058). Written informed consent was obtained from all patients.

Intraoperative CT images were obtained using an angiographic system (Artis Q; Siemens Healthcare, Germany) in a hybrid operating room. Under unilateral lung ventilation, each patient was placed in the lateral decubitus position, with the affected side facing upward. An axillary roll was placed under the thorax because the bed in the hybrid operation room could not be flexed. For C-arm rotation, both arms were placed on the side of the head and were secured by a side panel and some small pillows. The C-arm was placed at the caudal side of the operative field. Obese patients who could not fit into the $\mathrm{C}$-arm were excluded from IO-CT scans. Unlike conventional CT, a rotational $\mathrm{C}$-arm CT system has only limited scanning range. Therefore, we needed to use $\mathrm{C}$-arm fluoroscopy to augment the imaging range of IO-CT.

We placed 2-cm thoracoscopic ports at three locations: the fourth intercostal anterior axillary line, the seventh intercostal middle axillary line, and the seventh intercostal posterior axillary line.

We tried to locate tumors by finger palpation through the either thoracoscopic port. Intraoperative CT scans were performed after tumor marking with metal clips (Ligaclip MCA Medium, Ethicon Endo-surgery, Cincinnati, OH, USA). For difficult-to-palpate tumors, tumor marking was based on the anatomical position as follows (Figures 1,2). 

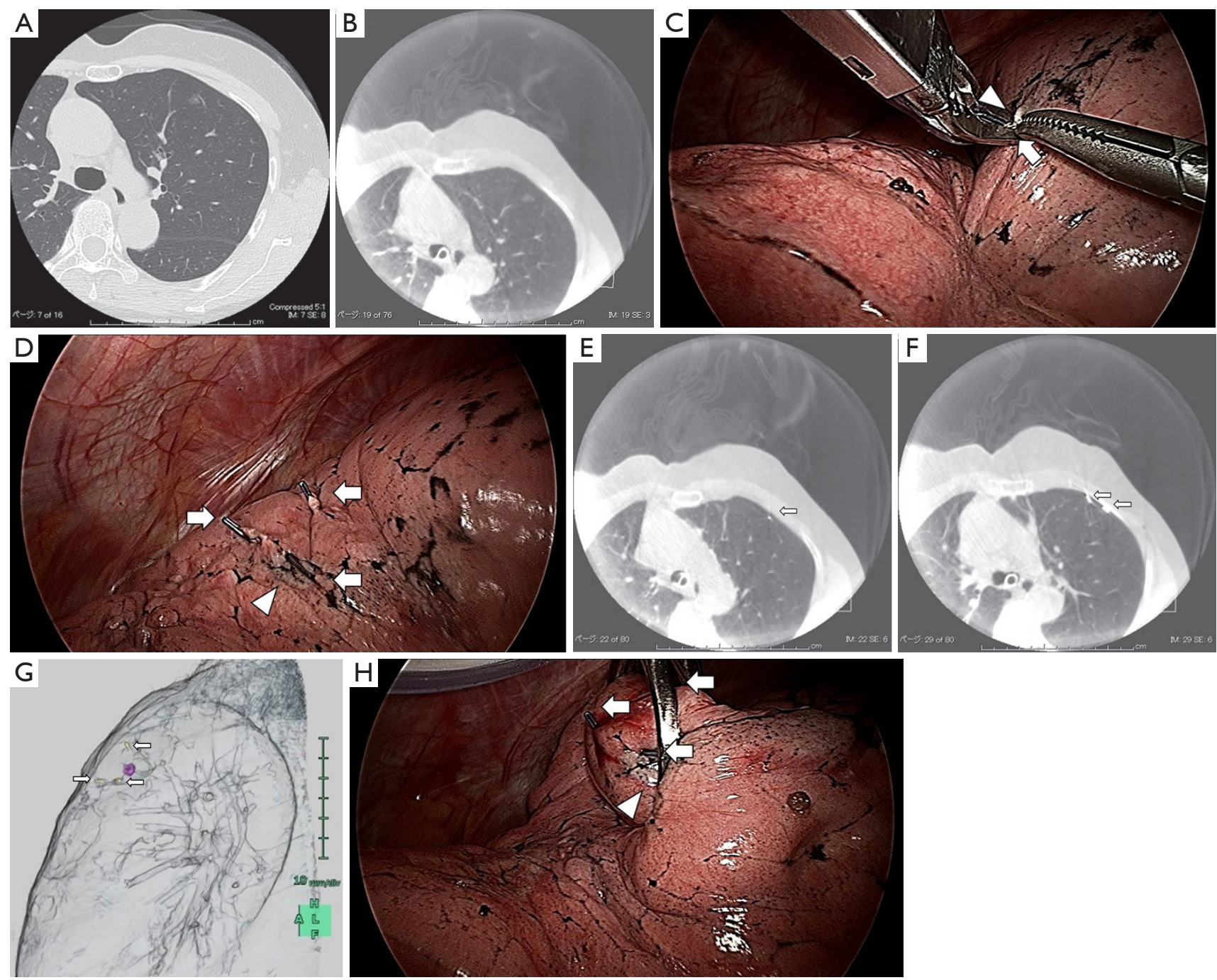

Figure 2 Pre-intraoperative CT and intraoperative pictures. (A) Preoperative computed tomography (CT) shows a pure grand-glass nodule in the left upper lobe; (B) intraoperative CT shows a pure grand-glass nodule that is comparable but slightly rougher than its preoperative CT counterpart image; (C,D) the surface of the visceral pleura around the SOFT COAG marking (arrowhead) was marked with metal clips (arrow); (E,F,G) intraoperative CT scans and 3D-CT imaging allowed us to find the tumor (in purple) and clips (arrow); (H) the tumor was held by ring forceps; wedge resection was performed using surgical staplers.

First, we estimated the intercostal muscle that could best be used as a marker (intercostal muscle just above the tumor, if possible) from preoperative CT. A needle was inserted along the caudal side of the intercostal muscle to make a slight "pin-point" depression in the parietal pleura. Next, under bilateral lung ventilation, the surface of the visceral pleura touching the parietal pleural needle depression was marked by a modern electrosurgical unit (VIO300D, ERBE Elektromedizin GmbH, Germany) in SOFT COAG mode, which could briefly draw a line on the visceral pleura without air leakage. Under unilateral lung ventilation again, metal clips were used to indicate the SOFT COAG marking. Intraoperative CT scans under bilateral lung ventilation and $3 \mathrm{D}-\mathrm{CT}$ imaging allowed us to locate the tumor by reference to the clips. At that time, by lining up both the preoperative and IO-CT images in one monitor, we could confirm that the target tumor did not differ in preoperative and IO-CT images.

When necessary, we could remove the first set of clips, re-clip the spot and perform a second IO-CT scan without 
Table 1 Clinical characteristics (study group, n=21)

\begin{tabular}{lc}
\hline Factors & $\mathrm{n}$ \\
\hline Age & $63.8 \pm 11.1$ [34-82] \\
Diagnosis & 10 \\
Lung cancer & 7 \\
Metastasis & 3 \\
Benign tumor & 1 \\
Hyperplasia & \\
Location & 11 \\
Right & 10 \\
Left & \\
Palpation & 12 \\
Palpable & 9 \\
Non-palpable & \\
\hline
\end{tabular}

injuring the visceral pleura. The tumor was held by ring forceps. We performed wedge resections during VATS, based on the marking clips, using surgical staplers.

\section{Statistical analysis}

The Mann-Whitney $U$ test was used to compare each factor. $\mathrm{P}<0.05$ was considered significant. Statistical analyses were performed with EZR (Saitama Medical Center, Jichi Medical University, Saitama, Japan), which is a graphical user interface for R (The R Foundation for Statistical Computing, Vienna, Austria). More precisely, it is a modified version of $\mathrm{R}$ Commander that is designed to add statistical functions frequently used in biostatistics (10).

\section{Results}

We employed this procedure for a total of 21 tumors in 18 patients, whose mean age was 63.8 years (range, 34-82 years). Of the 21 tumors, 11 were in the right lung and 10 in the left lung; 9 were non-palpable and 12 were palpable; in preoperative CT, 8 were seen as pure ground-glass nodule (GGN) type, 2 were partsolid type, and 11 were pure solid type; pathologically, 10 were primary lung cancers, 7 were metastatic lung cancers 3 were benign tumors, and 1 was hyperplasic (Tables 1,2). The tumors' mean size was $7.3 \pm$ $2.5 \mathrm{~mm}$, their mean solid area was $3.4 \pm 3.0 \mathrm{~mm}$, and their mean distance from the pleura was $6.8 \pm 5.1 \mathrm{~mm}$. Average solid area size for non-palpable tumors was significantly smaller $(0.9 \pm 1.8 \mathrm{~mm})$ than for palpable tumors $(5.3 \pm$ $2.1 \mathrm{~mm} ; \mathrm{P}<0.001)$; and average distance from the pleura was significantly longer for non-palpable tumors $(9.7 \pm 5.1 \mathrm{~mm})$ than for palpable tumors $(4.6 \pm 3.9 \mathrm{~mm} ; \mathrm{P}=0.022$; Table 3$)$.

Although the IO-CT image is slightly rougher than the preoperative $\mathrm{CT}$, all tumors were identified intraoperatively by using IO-CT scans (Figure 2A,B). All tumors were completely resected during VATS, with no intrapostoperative complications. Surgical margins were secured over the tumor diameters.

\section{Discussion}

Development of CT has improved detection of small lung tumors. As VATS procedures become more common, thoracic surgeons have increasing opportunities to resect lung tumors that are preoperatively non-palpable. Although some small lung tumors (such as pure GGN) might not require surgery on their own, when another lung tumor in the same side is indicated for surgery, we have to consider whether any small nearby lung tumor should also be resected. Even if a solid tumor is just below the visceral pleura, it may be difficult to palpate the tumor after freeing it from pleural adhesions.

According to the 2017 Lung Cancer stage classification, solid area size is more important than tumor total size for $\mathrm{T}$ factor $(11,12)$. Whether sublobar resection is indicated for small lung tumors (in terms of solid area) is the subject of several studies $(13,14)$. For these reasons, sublobar resection during VATS for small non-palpable lung tumors are increasingly considered necessary.

When lobectomy is performed, the target tumor (as recognized by preoperative $\mathrm{CT}$ ) is contained in the resected lung lobe. In addition, if lung lobulation is healthy, we needn't worry about the surgical margin. In contrast, the exact locations of both the tumor and the surgical margin are usually critical in sublobar resections.

In segmentectomy, we consider surgical margin to be a critical factor. Although the conventional ventilation and collapse method can identify the lung intersegmental plane, if surgical margin is insufficient, a nearby subsegment must be resected. Previously, we described how to establish anatomical intersegmental lines and obtain surgical margins with IO-CT scans during VATS segmentectomy (9). Because IO-CT scans can provide real-time lung images and wedge resection requires the exact tumor location, we 
Table 2 Patient characteristics (study group, $\mathrm{n}=21$ )

\begin{tabular}{|c|c|c|c|c|c|c|c|c|c|}
\hline Case & Age & Sex & Diagnosis & Location & $\begin{array}{l}\text { Tumor size } \\
\qquad(\mathrm{mm})\end{array}$ & $\begin{array}{l}\text { Solid size } \\
\quad(\mathrm{mm})\end{array}$ & $\begin{array}{l}\text { Distance from } \\
\text { the pleura }(\mathrm{mm})\end{array}$ & Tumor type & Palpation \\
\hline 1 & 63 & Female & Lung cancer & Rt.S6 & 10 & 0 & 7 & Pure GGN & Non-palpable \\
\hline 2 & 63 & Female & Lung cancer & Rt.S10 & 9 & 0 & 7 & Pure GGN & Non-palpable \\
\hline 3 & 42 & Female & Lung cancer & Rt.S5 & 8 & 0 & 8 & Pure GGN & Non-palpable \\
\hline 5 & 65 & Male & Benign tumor & Lt.S6 & 6 & 0 & 9 & Pure GGN & Non-palpable \\
\hline 6 & 65 & Male & Lung cancer & Lt.S10 & 5 & 5 & 10 & Solid & Non-palpable \\
\hline 7 & 66 & Female & Lung cancer & Lt.S1+2 & 5 & 0 & 12 & Pure GGN & Non-palpable \\
\hline 8 & 63 & Male & Metastasis & Rt.S8 & 10 & 3 & 22 & Part solid & Non-palpable \\
\hline 12 & 68 & Female & Benign tumor & Rt.S1 & 6 & 6 & 12 & Solid & Palpable \\
\hline 13 & 78 & Female & Lung cancer & Rt.S2 & 9 & 9 & 0 & Solid & Palpable \\
\hline 14 & 72 & Male & Metastasis & Rt.S10 & 5 & 5 & 0 & Solid & Palpable \\
\hline 15 & 74 & Female & Lung cancer & Lt.S6 & 5 & 5 & 5 & Solid & Palpable \\
\hline 16 & 70 & Female & Metastasis & Rt.S6 & 5 & 5 & 3 & Solid & Palpable \\
\hline 17 & 70 & Female & Metastasis & Rt.S8 & 6 & 6 & 8 & Solid & Palpable \\
\hline 18 & 57 & Female & Metastasis & Lt.S6 & 6 & 6 & 2 & Solid & Palpable \\
\hline
\end{tabular}

Table 3 Tumor information from preoperative CT: tumor size, solid area size and distance from the pleura

\begin{tabular}{lcccc}
\hline Tumor information & All tumor $(\mathrm{mm})$ & Non-palpable $(\mathrm{mm})$ & Palpable $(\mathrm{mm})$ & $\mathrm{P}$ value \\
\hline Tumor size & $7.3 \pm 2.5(5.0-14.0)$ & $8.2 \pm 2.9(5.0-14.0)$ & $6.6 \pm 2.0(5.0-11.0)$ & 0.178 \\
Solid area size & $3.4 \pm 3.0(0-9.0)$ & $0.9 \pm 1.8(0-5.0)$ & $5.3 \pm 2.1(0-9.0)$ & $<0.001$ \\
Distance from the pleura & $6.8 \pm 5.1(0-22.0)$ & $9.7 \pm 5.1(4-22.0)$ & $4.6 \pm 3.9(0-12.0)$ & 0.022 \\
\hline
\end{tabular}

consider that we could simplify this procedure to locate small non-palpable lung tumors with some precision.

After needle- and SOFT COAG marking were performed through the landmark intercostal muscle, according to preoperative CT readings, we used IO-CT scans with metal clips to obtain the exact location of the tumor.

Various methods have been used to locate lung microtumors, which are difficult to palpate. Although CT- guided short hook wires, lipiodol marking and coil marking have been used conventionally, such complications as pneumothorax, intrapulmonary hematoma, hemothorax, and air embolism have been reported (4). To avoid such complications, new techniques were reported recently. Sato et al. reported a bronchoscopic multispot dyemarking technique (VAL-MAP) (5). After preoperative bronchoscopic multispot dye marking was performed and 
VAL-MAP CT scans were reconstructed into 3D-CT, operation was performed according to the multiple dye spots. Toba et al reported on the efficacy of bronchoscopic metallic coil marking (6). A metallic coil was preoperatively installed, using ultrathin bronchoscopy. Afterwards, the patient underwent a wide wedge resection, using a C-arm-shaped roentgenographic fluoroscope during thoracoscopic surgery. In both procedures, preoperative bronchoscopic marking is necessary. However, dye or metallic markings can be far from the target tumor, because marker is installed in the bronchus.

Kondo et al. located GGNs with intraoperative ultrasonography (7). We consider this method to be the most useful and non-invasive without preoperative procedure. Unfortunately, as this method requires that patients not retain residual air, it is not appropriate for patients with chronic obstructive pulmonary disease or asthma with trapped air.

Kamiyoshihara et al. reported a method using a dyed swab technique (8). During preoperative CT, a special marker was used to identify the skin above the target tumor and dyed swab was used to mark just below the skin. Preoperative marking was performed under spontaneous negative pressure ventilation, whereas surgery was performed under positive pressure ventilation. We consider that this difference in ventilation mode can cause displacement of the marked location.

Our procedure resembles the Kamiyoshihara method, because our needle and SOFT COAG marking is based on the preoperative CT, which is performed under negative pressure ventilation in the dorsal position. Unlike their procedure, however, we added a metal clip marking and IO-CT scanning under positive pressure ventilation in the lateral decubitus position. Because IO-CT scans distribute real-time lung images, we can correct any misplacement and obtain the exact location of the tumor.

We had no intra-postoperative complications. Furthermore, we believe that air embolism cannot occur, because the visceral pleura is not damaged but only gripped by metal clips, which can be detached or moved without injuring the visceral pleura.

This procedure can also be used for tumors near the mediastinum or diaphragm. As no intercostal muscle landmarks are near the mediastinum or diaphragm when we first make the initial SOFT COAG and metal clip markings, we may be far from the tumor. However, as IO-CT shows the location of the tumor and metal clips, we can re-clip and re-scan to obtain a more accurate tumor location.

In this study, we performed IO-CT scans for patients who, preoperatively, we thought had non-palpable lung tumors. As non-palpable tumors are generally significantly smaller in solid area size, and farther from the pleura, than are palpable tumors, we consider that IO-CT is indicated for patients with non-palpable tumors. However, as no clear guidelines exist, surgeons who are concerned about whether tumors can be palpated intraoperatively might "err on the side of caution" by using IO-CT.

This procedure has several limitations. First, the distance between the visceral pleura and the tumor is initially unknown. Metallic clips are not marked in the lung parenchyma, but on the surface of the visceral pleura. In the hook-wire marking system, the hook wire is marked near the tumor in the lung parenchyma; if the hook wire is in the resected specimen, the tumor will be removed (1). Although the entire tumor could logically be located by its collective vertical, horizontal and depth distances from the landmark, in our procedure, vertical distance between the visceral pleura and the tumor must be calculated from preoperative or IO-CT scans.

Second, the CT device is necessary to identify the metal clips and tumor in this procedure. Therefore, this method cannot be used at every facility. However, in the cardiac, vascular, and neurosurgery fields, hybrid operating rooms with IO-CT scans are commonly accessible. In our hospital, IO-CT scans are used not only in the above fields, but also in repairing facial or pelvic fractures. As hybrid operating rooms and IO-CT scans are useful in a wide range of procedures, they will become increasingly available.

Finally, patients' radiation exposure is a consideration. However, radiation exposure from intraoperative chest CT scans are reportedly within the range of low-dose CT scans for lung cancer screening $(15,16)$. We therefore think that radiation exposure for this technique is acceptable.

In conclusion, IO-CT scans after tumor marking with metal clips during VATS can help precisely locate small non-palpable lung tumors near the mediastinum or diaphragm, using this simple and feasible method. IO-CT scans should be indicated for tumors that are preoperatively considered to be non-palpable, particularly tumors with small solid areas or those located far from the pleura.

\section{Acknowledgements}

None.

\section{Footnote}

Conflicts of Interest: The authors have no conflicts of interest 
to declare.

Ethical Statement: The study protocol was approved by the Institutional Review Board of Kochi Health Science Center, Japan (No. 141058) and written informed consent was obtained from all patients.

\section{References}

1. Dendo S, Kanazawa S, Ando A, et al. Preoperative localization of small pulmonary lesions with a short hook wire and suture system: experience with 168 procedures. Radiology 2002;225:511-8.

2. Nomori H, Horio H, Naruke T, et al. Fluoroscopyassisted thoracoscopic resection of lung nodules marked with lipiodol. Ann Thorac Surg 2002;74:170-3.

3. Lizza N, Eucher P, Haxhe JP, et al. Thoracoscopic resection of pulmonary nodules after computed tomographic-guided coil labeling. Ann Thorac Surg 2001;71:986-8.

4. Kamiyoshihara M, Sakata K, Ishikawa S, et al. Cerebral arterial air embolism following CT-guided lung needle marking. Report of a case. J Cardiovasc Surg (Torino) 2001;42:699-700.

5. Sato M, Yamada T, Menju T, et al. Virtual-assisted lung mapping: outcome of 100 consecutive cases in a single institute. Eur J Cardiothorac Surg 2015;47:e131-9.

6. Toba H, Kondo K, Miyoshi T, et al. Fluoroscopy-assisted thoracoscopic resection after computed tomographyguided bronchoscopic metallic coil marking for small peripheral pulmonary lesions. Eur J Cardiothorac Surg 2013;44:e126-32.

7. Intraoperative ultrasonographic localization of pulmonary ground-glass opacities. Kondo R, Yoshida K, Hamanaka K, et al. J Thorac Cardiovasc Surg 2009;138:837-42.

8. Kamiyoshihara M, Ibe T, Kawatani N, et al. A convenient method for identifying a small pulmonary nodule using a dyed swab and geometric mapping. J Thorac Dis 2016;8:2556-61.

9. Chang SS, Okamoto T, Tokunaga Y, et al. Intraoperative computed tomography navigation during thoracoscopic segmentectomy for small-sized lung tumors. Semin Thorac Cardiovasc Surg 2017; S1043-0679:30221-6.

10. Kanda Y. Investigation of the freely available easy-touse software 'EZR' for medical statistics. Bone Marrow Transplant 2013;48:452-8.

11. Rami-Porta R, Bolejack V, Crowley J, et al. The IASLC lung cancer staging project: proposals for the revisions of the $\mathrm{T}$ descriptors in the forthcoming eighth edition of the TNM Classification for Lung Cancer. J Thorac Oncol 2015;10:990-1003.

12. Detterbeck FC, Boffa DJ, Kim AW, et al. The eighth edition lung cancer stage classification. Chest 2017;151:193-203.

13. Nakamura K, Saji H, Nakajima R, et al. A phase III randomized trial of lobectomy versus limited resection for small-sized peripheral non-small cell lung cancer (JCOG0802/WJOG4607L). Jpn J Clin Oncol 2010;40:271-4.

14. Aokage K, Saji H, Suzuki K, et al. A non-randomized confirmatory trial of segmentectomy for clinical T1N0 lung cancer with dominant ground glass opacity based on thin-section computed tomography (JCOG1211). Gen Thorac Cardiovasc Surg 2017;65:267-72.

15. Gill RR, Zheng Y, Barlow JS, et al. Image-guided video assisted thoracoscopic surgery (iVATS)-phase I-II clinical trial. J Surg Oncol 2015;112:18-25.

16. Abu Saleh WK, Jabbari OA, Lumsden A, et al. Case Report: Simultaneous Localization and Removal of Lung Nodules Through Extended Use of the Hybrid Suite. Methodist Debakey Cardiovasc J 2015;11:245-6.
Cite this article as: Tokunaga Y, Chang SS, Kita Y, Okamoto T. Intraoperative computed tomography after tumor marking with metal clips for non-palpable lung tumors. J Thorac Dis 2018;10(7):4094-4100. doi: 10.21037/jtd.2018.06.123 